\title{
Is prenatal genomic testing ready for prime time?
}

\author{
Margaret P. Adam, MD, MS $\mathrm{S}^{1,2}$
}

Genomic testing, including exome sequencing, genome sequencing, and mitochondrial sequencing, is used in postnatal clinical settings to identify the genetic basis of undiagnosed findings in a symptomatic individual. ${ }^{1-3}$ Such studies have a diagnostic yield of $25-40 \%{ }^{4}$ Given the diagnostic utility of genomic testing in a postnatal setting, it is inevitable that genomic testing will eventually be incorporated into the prenatal realm.

The article by Boissel et al. ${ }^{5}$ entitled "Genomic Study of Severe Fetal Anomalies and Discovery of GREB1L Mutations in Renal Agenesis" in this issue of Genetics in Medicine explores the clinical utility of whole-exome sequencing (WES) when applied to 101 stillborn or terminated fetuses with congenital anomalies (at least two major malformations; a structural brain malformation and/or severe ventriculomegaly; or an anomaly in which postnatal lethality is likely). The authors hypothesize that de novo pathogenic variants account for a substantial proportion of severe fetal phenotypes that may not be represented in postnatal populations due to the serious nature of the findings. In their cohort, karyotype and/ or chromosomal microarray analysis was performed without the identification of a cause for the fetal features and 85 fetuses also underwent autopsy. WES was then used to determine if an underlying genetic cause could be found. In 18 cases proband-only WES was done due to a suspected autosomal recessive condition (presence of parental consanguinity, more than one affected sibling, or fetal features suggesting a known autosomal recessive condition, such as a ciliopathy). For the remaining 83 cases, trio-WES was completed. In this cohort the authors only considered rare de novo variants or variants in known disease genes thought to be associated with the postmortem phenotype. In their search for novel disease genes, the authors chose candidate genes based on their function in model organisms or by their discovery in fetuses with similar phenotypes from unrelated families. In 19 cases a molecular diagnosis was identified, although in 13 cases the diagnosis was not suspected because the fetal features were nonspecific, more severe, or significantly different from what has been reported in the literature for the known condition. Furthermore, novel candidate genes associated with perinatal lethality were discovered.
This study and others like it that assess a prenatal cohort in a postmortem setting are the first step toward helping clinicians understand both the power and the problems with genomic testing when applied in a prenatal setting. Similar to the implementation of other diagnostic technologies into clinical practice, the issues associated with prenatal genomic testing are not new. When chromosomal microarray technology was first applied in a postnatal clinical setting, issues related to the interpretation of copy-number variants and, in particular, variants of unknown clinical significance were debated. As chromosomal microarray became standard of care in a postnatal setting, ${ }^{6}$ practitioners considered under what circumstances it should be used prenatally. Initially, chromosomal microarray testing was offered in the context of a fetus with multiple anomalies and a documented normal karyotype. The platform used in the prenatal setting was initially restricted to known pathogenic copy-number variants to avoid the finding of a variant of unknown significance in an ongoing pregnancy. However, within a few years, these informal guidelines fell by the wayside and now singlenucleotide polymorphism chromosomal microarray is frequently offered to any pregnant woman who wants to assess for a chromosome abnormality in her fetus.

To date, the highest diagnostic yield for prenatal WES has been in fetuses with multiple anomalies or specific types of anomalies, such as structural brain malformations, ${ }^{7,8}$ with diagnostic rates that are similar to those reported in the study by Boissel et al. ${ }^{5}$ Furthermore, trio testing has a slightly higher diagnostic yield compared with proband-only testing, particularly for sporadic fetal findings in the absence of parental consanguinity or a family history of similar findings. Such cases are highly selected with a greater a priori risk that the fetus has a single-gene condition.

Comprehensive genetic counseling for prenatal genomic testing is critical. Even in selected populations of families who are well-educated about genetic testing options in pregnancy there is a tendency for families to view genomic testing as a means to confirm that their fetus will be "normal." current WES technology is unable to detect certain genetic changes (e.g., copy-number variants at a single-exon level that may be undetectable by single-nucleotide polymorphism array, nucleotide repeat expansions, epigenetic changes) and

${ }^{1}$ Division of Genetic Medicine, Department of Pediatrics, University of Washington, Seattle, Washington, USA; ${ }^{2}$ Seattle Children's Hospital, Seattle, Washington, USA.

Correspondence: Margaret P. Adam (margaret.adam@seattlechildrens.org)

Advance online publication 22 March 2018. doi:10.1038/gim.2018.5 
is not as accurate as Sanger sequencing, pathogenic variants can be missed. Therefore, parental expectations about the utility of WES must be managed appropriately.

In a postnatal setting, complete phenotyping, including inspection for subtle dysmorphic features and information about developmental progress, is possible, while such precise phenotyping is limited in a prenatal setting. Furthermore, laboratories that perform genomic testing use algorithms for variant interpretation that rely on the phenotypic information they are given. In the situation of either an incomplete phenotype or a phenotype that diverges from the classic syndrome, diagnostic accuracy may be lost. In the study by Boissel et al. $^{5}$ a fetus with a de novo pathogenic EP300 truncating variant had spina bifida and postaxial polydactyly on ultrasound with the dysmorphic features of RubensteinTaybi syndrome (RTS) identified at autopsy. Without being able to assess for the recognizable facial and limb findings of RTS, a clinician who receives this result in a prenatal setting may not know how to counsel the family and would be concerned that the fetus might have a secondary diagnosis that explains the malformations in addition to RTS. If the variant identified in EP300 happened to be a previously unreported missense variant, a possible diagnosis of RTS may have been overlooked. Many clinical laboratory reports use a statement to the effect of "clinical correlation is required," which leaves the clinician to synthesize the genomic results with a limited phenotype and attempt to render a conclusion that is helpful to families who are in the midst of making important pregnancy decisions.

Unlike the initial implementation of targeted chromosomal microarray into prenatal testing, it may be more difficult to target genomic testing to solely known pathogenic variants in known disease-causing genes. This will certainly decrease diagnostic yield, particularly for those conditions caused by pathogenic missense variants that have not been reported previously or pathogenic variants in novel disease genes, such as Boissel et al. ${ }^{5}$ found with GREB1L.

Prenatal genomic testing is also plagued by practical concerns as reviewed by Best et al., ${ }^{7}$ such as availability of genetics professionals who can provide pre- and posttest genetic counseling, price of the test, turnaround time (most women do not undergo an extensive anatomy ultrasound until 18-20 weeks gestation), disclosure of secondary or incidental findings, availability of both biological parents to provide samples, and education of nongenetics providers about the strengths and limitations of the testing. The overall cost of implementing prenatal genomic testing has not been systematically studied, but must take into account more than just the price of the test itself. For example, infrastructure to store large amounts of genomic data, further confirmatory testing with resulting health surveillance for secondary findings, and need for more comprehensive genetic counseling by trained genetics professionals are some of the hidden costs. ${ }^{7}$ How receptive insurance companies will be to covering prenatal genomic testing remains to be seen.

Genomic testing is clearly a powerful tool that augments traditional autopsy and cytogenetic studies to determine the etiology of significant, often multiple, malformations in stillborn or terminated fetuses. It will most certainly expand our understanding of the causes of severe or lethal genetic conditions that present in the prenatal period. However, applying this tool to a current ongoing pregnancy in which nonspecific fetal ultrasound markers are found or in a routine setting in which the a priori risk of the fetus having an anomaly or single-gene disorder is low may lead to uninterpretable findings or, alternatively, provide a false sense of security that all genetic syndromes have been "ruled out." The finding of a variant of unknown clinical significance or fetal features that are incongruent with prenatal genomic results poses a significant ethical issue in an ongoing pregnancy where a couple is actively making decisions about continuing or interrupting a pregnancy. However, genomic testing may be appropriate when multiple anomalies or possible lethal findings are identified prenatally in a euploid fetus after the family undergoes comprehensive genetic counseling that addresses the limitations of the testing and potential for secondary findings. ${ }^{10}$

\section{DISCLOSURE}

The author declares no conflict of interest.

\section{REFERENCES}

1. Gahl WA, Markello TC, Toro C, et al. The National Institutes of Health undiagnosed diseases program: insights into rare diseases. Genet Med 2012:14:51-59.

2. Manolio TA, Chisholm RL, Ozenberger B, et al. Implementing genomic medicine in the clinic: the future is here. Genet Med 2013;15:258-267.

3. Biesecker LG \& Green RC. Diagnostic clinical genome and exome sequencing. N Engl J Med 2014;370:2418-2425.

4. Sawyer SL, Hartley T, Dyment DA, et al. Utility of whole-exome sequencing for those near the end of the diagnostic odyssey: time to address gaps in care. Clin Genet 2016;89:275-284.

5. Boissel S, Fallet-Bianco C, Chitayat D, et al. Genomic study of severe fetal anomalies and discovery of GREB1L mutations in renal agenesis. Genet Med 2018;20:XXX-XXX.

6. Miller DT, Adam MP, Aradhya $S$, et al. Consensus statement: chromosomal microarray as a first-tier clinical diagnostic test for individuals with developmental disabilities or congenital anomalies. Am J Hum Genet 2010;86:749-764.

7. Best $S$, Wou K, Vora N, et al. Promises, pitfalls and practicalities of prenatal whole exome sequencing. Prenat Diagn; e-pub ahead of print 27 June 2017

8. Fu F, Li R, Li Y, et al. Whole exome sequencing as a diagnostic adjunct to clinical testing in a tertiary referral cohort of 3988 fetuses with structural anomalies. Ultrasound Obstet Gynecol; e-pub ahead of print 4 October 2017.

9. Quinlan-Jones E, Hillman SC, Kilby MD \& Greenfield M. Parental experiences of prenatal whole exome sequencing (WES) in cases of ultrasound diagnosed fetal structural anomaly. Prenat Diagn 2017;37: $1225-1231$.

10. Vora NL, Powell B, Brandt A, et al. Prenatal exome sequencing in anomalous fetuses: new opportunities and challenges. Genet Med 2017;19:1207-1216. 